\title{
8 Wie finde ich einen geeigneten Operateur und eine gute Klinik?
}

Grundsätzlich gilt: Eine hohe Behandlungsfrequenz einer Klinik und eines Operateurs bei einer bestimmten operativen Prozedur sichert mit höherer Wahrscheinlichkeit ein gutes Ergebnis.

Sie brauchen daher einen Arzt, der sich langjährig mit der Schulter beschäftigt hat, daneben aber den gesamten Bewegungsapparat überblickt und auch Krankheitsbilder aus benachbarten Fachgebieten abgrenzen kann. Dies kann, muss aber beileibe nicht immer der Chefarzt einer Klinik sein. Oft hat beispielsweise der Oberarzt mehr Routine durch seine tägliche Präsenz im Operationssaal. Die Politik hat aus diesem Grunde Mindestfallzahlen in einigen Bereichen eingeführt. Auch geben spezielle Zertifizierungen (z. B. Endoprothesenzentrum) Auskunft über bestandene Qualitätsaudits der Ärzte und Kliniken. Fragen Sie den potentiellen Operateur direkt, wie viel Erfahrung er speziell in Ihrem Fall hat!

Aber nicht nur der Arzt als Operateur ist entscheidend; das gesamte Team, das gut aufeinander abgestimmt ist und über eine große Routine verfügt, garantiert ein gutes Ergebnis. Schließlich sollte auch die Infrastruktur des Krankenhauses alles Notwendige bieten, wie z. B. modern ausgestattete postoperative Überwachungseinheiten (ICU = Intermediate Care Unit).

Bei der Auswahl der Klinik können Patientenforen und Klinikbewertungsportale im Internet hilfreich sein, die Bewertungen sind jedoch subjektiv und keinesfalls repräsentativ. Die wohl verlässlichsten Informationen bieten die von den Kliniken veröffentlichten Qualitätsberichte.

\section{WebTipp}

Hilfe bei der Kliniksuche erhalten Sie z. B. unter

www.tk.de/tk/klinikfuehrer/114928 oder

www.weisse-liste.de.

R. Kipping, Operation Schulter, DOI 10.1007/978-3-89935-300-6_8,

(C) Springer Medizin Verlag GmbH 2016 